\title{
Statistical Tests for Evaluating Earthquake Prediction Methods
}

\author{
Kurt S. Riedel \\ Courant Institute of Mathematical Sciences \\ New York University
}

\begin{abstract}
The impact of including postcursors in the null hypothesis test is discussed. Unequal prediction probabilities can be included in the null hypothesis test using a generalization of the central limit theorem. A test for determining the enhancement factor over random chance is given. The seismic earthquake signal may preferentially precede earthquakes even if the VAN methodology fails to forecast the earthquakes. We formulate a statistical test for this possibility.
\end{abstract}

\section{Postcursors and High Significance Level}

The present paper by Varotsos et al. [1996, hereafter cited as VEVL] is devoted primarily to criticizing the criticism of Mulargia and Gasperini [1992, herafter cited as MG], although this paper does contain some clarification of the methodology of Varotsos et al. [1981, hereafter cited as VAN]. The central argument is whether the VEVL method predicts better than random chance. If the null hypothesis is true, the significance level is a random variable with a mean value of $1 / 2$ and is uniformly distributed on $[0,1]$. Normally, one requires significance levels as low as 0.05 before one is confident that the random chance hypothesis is false.

Many of Mulargia and Gasperini's tests achieve a significance level of .999, which means that under the MG test, VAN do much worse than the hypothesis of random chance would expect. There are three possible explanations for the significance level of .999: 1) VAN are extremely unlucky; 2) VAN predictions are anticorrrelated with earthquakes; 3) the MG hypothesis that earthquakes are independent and uniformly distributed in time is wrong. Point 5 of the VEVL criticism suggests the likely culprit, i.e., many of MG's earthquakes are probably aftershocks which VAN did not try to predict. Note that the significance level of the test will converge to 1.0 as the number of predictions tends to infinity if the null hypothesis of random chance is true and a small percentage of the spatial distribution of earthquakes are postcursors which VAN do not try to predict. Thus the MG test should be repreated after excluding these postcursors. It is unfortunate that VEVL did not formulate a criterion for excluding postcursors. 


\section{Spatial Distribution of Earthquakes}

MG do not include the limited spatial extent of the VAN predictions in their analysis. Thus, MG are testing only the temporal accuracy and not the spatial accuracy of the predictions. Such spatially averaged tests cannot exclude the possibility that VAN can accurately predict the location of the next earthquake, but has little success predicting the time of occurence. Nevertheless, testing the temporal accuracy is of interest.

The alternative, including the spatial extent of the VAN predictions as Takayama [1993] does, bears the risk that earthquakes are clustered on faults and that VAN are essentially predicting occurences on the fault lines. By spatially averaging, the MG analysis is robustified against the spatial distribution of earthquakes. In contrast, Takayama's analysis could depend quite sensitively on the spatial distribution of earthquakes.

Since I am not a specialist, my only knowledge of the distribution of Greek earthquakes is from Fig. 19 of Varotsos and Lazaridou [1991, hereafter cited as VL]. This figure makes the earthquake distribution look highly nonhomogeneous, centered on the island of Kefnalonia and running NW to SE.

In estimating the spatial distribution of earthquakes, the entire record of Greek earthquakes could be used i.e., earthquakes which occured before the VAN system was implemented could be used. Including these earlier earthquakes assumes that the spatial distribution of earthquakes has not changed appreciably over the time record. In their response, I urge VEVL to plot both the distribution of Greek earthquakes over the entire time record and over the subrecord on which the VAN system was making predictions. It would also be interesting to see the spatial distribution of VAN predictions side by side.

From Fig. 19 of VL, a reasonable model for the spatial probability is

$$
p(\vec{x})=p_{0}+p_{1} \exp \left(\left(\vec{x}-\vec{x}_{c}\right)^{\dagger} Q\left(\vec{x}-\vec{x}_{c}\right)\right),
$$

where $\vec{x}_{c}$ is the most likely location of an earthquake, $Q$ is a $2 \times 2$ positive definite matrix and $p_{0}$ is the spatially homogeneous component of the distribution. Given the constraint that $\int p(x) d x=1$, this distribution has six free parameters. These parameters may be estimated by maximizing the log-likelihood: $\sum_{i=1}^{N} \log \left[p\left(\vec{x}_{i}\right)\right]$ where $\vec{x}_{i}$ is the location of the $i$ th earthquake. Alternatively, the density can be estimated using bivariate kernel smoothers. (See Silverman [1986] and Scott [1992] for reviews.)

\section{Null Hypothesis Testing with Unequal Probabilities}

Including the spatial inhomogeneity and the varying temporal lengths of the predictions makes the probability of a correct prediction due to chance, $j=1 \ldots M$, nonuniform. Let $p_{j}$ be the probability that the $j$-th forecast is correct due to random chance. The nonuniformity makes the Poisson summation formula nonapplicable. To evaluate the significance level exactly is very computationally intensive. Monte-Carlo evaluations are also costly. Instead, we recommend using Lindeberg's generalization of the central limit theorem Lamperti [1966, p. 69]. In our context, it states that

$$
\frac{\left(X_{M}-\sum_{j=1}^{M} p_{j}\right)}{\sqrt{\sum_{j=1}^{M} p_{j}\left(1-p_{j}\right)}} \stackrel{M \rightarrow \infty}{\longrightarrow} N(0,1)
$$

where the random variable, $X_{M}$, is the number of correct predictions out of $M$ independent random attempts. We let $N_{M}$ be the number of correct predictions in the present data set. Using these asymptotics, the probability that $X_{M}$ is greater than or equal to $N_{M}$ is then approximated by

$$
P\left(N_{M} \leq X_{M}\right)=
$$




$$
P\left(N_{M}-\frac{1}{2} \leq X_{M}\right) \simeq 1-\Phi\left(\frac{N_{M}-\mu_{M}-\frac{1}{2}}{\sigma_{M}}\right)
$$

where

$$
\mu_{M} \equiv \sum_{j=1}^{M} p_{j}, \quad \sigma_{M}^{2} \equiv \sum_{j=1}^{M} p_{j}\left(1-p_{j}\right),
$$

and $\Phi$ is the cumulative error function. Since $\mu_{M} / \sigma_{M}$ increases as $\sqrt{M}$, this test will yield significance levels near zero as $M \rightarrow \infty$ even if the VAN method is only slightly better than random chance.

A more sophisticated question is: by what factor, $c$ is the VAN method better than random chance? Here, $c=2$ means than the VAN success probability is twice as large as random chance. The enhancement factor can be estimated by

$$
\begin{aligned}
\hat{c}=N_{M} / \mu_{M}= & \text { number predicted over number } \\
& \text { predicted by random chance } .
\end{aligned}
$$

A more skeptical person would like to require a $2 \sigma$ confidence interval on the estimate of the enhancement factor. In other words, he/she would not accept the maximum likelihood estimate of $c$, but instead choose the smallest value of $c$ that is consistent with the data. Suppose the VAN predictive probability were $p_{j}^{(\mathrm{VAN})}=c p_{j}^{(\text {Random })}$, and $\mu^{(\mathrm{VAN})}=c \mu_{M}^{(\mathrm{Random})}$ and $\sigma_{c}^{(\mathrm{VAN})}=\sum_{j=1}^{M} c p_{j}\left(1-c p_{j}\right)$. We assume that $c p_{j}^{(\text {Random })} \leq 1$ for each value of $p_{j}$. We define the VAN predictive inflation factor as the minimal value of $c$ such that the desired significance level (such as 0.05 ) equals

$$
P_{c}\left(X_{M} \geq N_{M}-\frac{1}{2}\right)=1-\Phi\left(\frac{N_{M}-c \mu_{M}-\frac{1}{2}}{\sigma_{c}}\right) .
$$

When $p_{j}<\frac{1}{2}$, this probability inflation factor is less than the simple ratio of $X_{M} / \mu^{\text {(Random) }}$.

\section{A Test for Precursor Activity}

It is possible that the seismic earthquake signals (SES) do tend to precede earthquakes, but that the VAN algorithm does not forecast them well. In particular, the temporal forecast might be inaccurate. Thus we now change the question from "Is VAN better than random chance?" to the more general question "Do SES tend to precede earthquakes preferentially?" Thus we test only whether the SES are earthquake precursors on average and we do not test the temporal accuracy of the VAN forecast.

For simplicity, we neglect spatial dependencies and consider the time, $T$, between the first prediction and the last earthquake. We assume that there are $M$ predictions and $N$ earthquakes. We define $\tau_{j}$ to be the time between the $j$-th prediction and the next earthquake. Under the assumption that the first $N-1$ earthquakes are uniformly distributed in time, $\tau_{j}$ is distributed as $p\left(\tau_{j} \mid t_{j}\right)=(N-1)\left(1-\frac{\tau_{j}}{T}\right)^{N-2} / T$ for $0 \leq \tau_{j} \leq T-t_{j}$ and $P\left(\tau_{j}=T-t_{j}\right)=\left(\frac{t_{j}}{T}\right)^{N-1}$, where $t_{j}$ is the time of the $j$-th prediction. We use the test statistic $Y_{M}=\sum_{j=1}^{M} \tau_{j}$. The expectation of $Y_{M}=\sum_{j=1}^{M} E\left[\tau_{j}\right]=\frac{T}{N} \sum_{j=1}^{M}\left[1-\left(\frac{t_{j}}{T}\right)^{N}\right]$. The variance of $\tau_{j}$ is

$$
\begin{aligned}
\operatorname{Var}\left[\tau_{j}\right]= & T^{2}\left[\frac{N-1}{N^{2}(N+1)}\right. \\
& \left.+\frac{2}{(N+1)}\left(\frac{t_{j}}{T}\right)^{N+1}+\frac{1}{N^{2}}\left(\frac{t_{j}}{T}\right)^{2 N}\right]^{2},
\end{aligned}
$$


but the $\tau_{j}$ are not independent. If we assume that $t_{j}, j=2, \ldots, M$, are independently distributed in $[0, T]$ and average over the distribution of $t_{j}$, the $\tau_{j}$ become independent. In this case, we can apply the central limit theorem using $\operatorname{Var}\left[Y_{M}\right]=\sum_{j=1}^{M} \operatorname{Var}\left[\tau_{j} \mid t_{j}\right]$. Let $\hat{\tau}_{j}$ be the observed delay time between the $j$ prediction and the next earthquake. If $\Sigma \hat{\tau}_{j}-E\left[Y_{M}\right]$ is much smaller than $-2.5 \sqrt{\operatorname{Var}\left[Y_{M}\right]}$, then the SES signals occur preferentially before earthquakes. A similar test can be used to test whether the SES signals tend to occur after earthquakes. We caution that this test will be sensitive to the clustering of earthquakes and predictions into high activity time periods. If VEVL systematically exclude SES measurements which occur immediately after an earthquake and the SES measurements are otherwise randomly distributed, this test will confirm that the recorded SES signals occur preferentially before an earthquake.

\section{Risk Analysis and the VEVL Paradox}

This nonparametric test may be of value due to the ad hoc nature of the VAN algorithms in predicting the spatial and temporal region where an earthquake might occur. I strongly suspect that more detailed statistical analysis could improve the forecasting algorithm given SES measurements at various spatial locations.

The final topic which I would like to address is the paradox in the appendix of VEVL. The issue is: how does the uncertainty of predicting the earthquake amplitude affect the hypothesis testing? VEVL's appendix raises more general issues such as the relative loss of predicting an earthquake which does not occur (or occurs at low amplitude) versus not forecasting a large amplitude quake. A forecast is an action taken under uncertainty, and an incorrect forecast will cause an economic cost. To truly assess the effectiveness of an earthquake prediction scheme, the loss associated with the scheme and the resulting policy decisions needs to be compared with the costs of ignoring the prediction. When a realistic assessment of the loss associated with predicting an earthquake which does not occur is made, I believe that many prediction schemes will be economically useless, but statistically significant.

Acknowledgments. The helpful comments of Dr. Geller and Dr. Vallianatos are acknowledged. This work was supported by U.S. Department of Energy Grant No. DE-FG02-86ER53223.

\section{References}

Lamperti, J., Probability: A Survey of the Mathematical Theory, Benjamin/Cummings, Reading, Massachusetts, 1966.

Mulargia, F., and P. Gasperini, Evaluating the statistical validity beyond chance of VAN earthquake precursors, Geophys. J. Int., 111, 33-44, 1992.

Scott, D. W., Multivariate Density Estimation, John Wiley, New York, 1992.

Silverman, B.-W., Density Estimation for Statistics and Data Analysis, Chapman-Hall, New York, 1986.

Takayama, H., Comment on Evaluating the statistical validity beyond chance of "VAN" earthquake precursors,' Geophys. J. Int., 115, 1197-1198, 1993.

Varotsos, P., K. Alexopoulos, and K. Nomicos, Seismic electric currents, Prakt. Akad. Athenon, 56, 277-286, 1981.

Varotsos, P., K. Eftaxias, F. Vallianatos, and M. Lazaridou, Basic principles for evaluating an earthquake prediction method, Geophys. Res. Lett., this issue, 1996.

Varotsos, P., and M. Lazaridou, Latest aspects of earthquake prediction in Greece based on seismic electric sig- 
nals, Tectonophysics, 188, 321-347, 1991.

(Received May 21, 1994; revised September 15, 1994; accepted October 31, 1995.)

RIEDEL: STATISTICAL TESTS FOR EARTHQUAKE PREDICTION RIEDEL: STATISTICAL TESTS FOR EARTHQUAKE PREDICTION RIEDEL: STATISTICAL TESTS FOR EARTHQUAKE PREDICTION RIEDEL: STATISTICAL TESTS FOR EARTHQUAKE PREDICTION RIEDEL: STATISTICAL TESTS FOR EARTHQUAKE PREDICTION RIEDEL: STATISTICAL TESTS FOR EARTHQUAKE PREDICTION RIEDEL: STATISTICAL TESTS FOR EARTHQUAKE PREDICTION RIEDEL: STATISTICAL TESTS FOR EARTHQUAKE PREDICTION RIEDEL: STATISTICAL TESTS FOR EARTHQUAKE PREDICTION RIEDEL: STATISTICAL TESTS FOR EARTHQUAKE PREDICTION 